\title{
Specific Inhibition of the Polymorphonuclear Leukocyte Chemotactic Response to Hydroxy-Fatty Acid Metabolites of Arachidonic Acid by Methyl Ester Derivatives
}

\author{
Edward J. Goetzl, Frank H. Valone, Vernon N. Reinhold, and Robert R. \\ GoRMAN, Howard Hughes Medical Institute Laboratory at Harvard Medical \\ School, Departments of Medicine, Harvard Medical School, and Robert B. \\ Brigham Hospital Division of the Affiliated Hospitals Center, the Laboratory of \\ Human Reproduction and Reproductive Biology, Harvard Medical School, \\ Boston, Massachusetts 02115; The Upjohn Company, Kalamazoo, Michigan 49001
}

\begin{abstract}
A B S T RACT The human polymorphonuclear (PMN) leukocyte chemotactic activity of the hydroxy-fatty acid metabolites of arachidonic acid, 12-L-hydroxy-5,8,10heptadecatrienoic acid (HHT) and 12-L-hydroxy5,8,10,14-eicosatetraenoic acid (HETE), is eliminated by methylation. Both methyl esters are specific competitive inhibitors of the PMN leukotactic responses to the parent stimuli, and exert no effect on the responses to formyl-methionyl peptides or chemotactic fragments of the fifth component of complement. 50\% inhibition of the in vitro chemotactic responses of PMN leukocytes to HETE and HHT was achieved by an equimolar concentration of the corresponding methyl esters, whereas reciprocal cross-inhibition was observed at molar ratios of HETE methyl ester to HHT and HHT methyl ester to HETE which reflected the three- to fivefold greater chemotactic potency of HETE relative to HHT. Methyl esters of structurally related, but nonchemotactic, fatty acids did not competitively inhibit the chemotaxis elicited by HETE or HHT. The intraperitoneal injection of HETE in guinea pigs evoked an eosinophil response at $30 \mathrm{~min}$ and a neutrophil response at $5 \mathrm{~h}$, which were prevented by a one- to twofold molar ratio of HETE methyl ester. The competitive inhibition of the in vitro chemotactic activity and the in vivo leukotactic effect of the unsaturated hydroxy-fatty acids by homologous methyl ester derivatives suggests that the cellular component of natural inflammatory reactions may be susceptible to specific regulation by receptor-directed modulation of the activity of the predominant chemotactic principles.
\end{abstract}

Received for publication 24 November 1978 and in revised form 26 January 1979.

\section{INTRODUCTION}

Specific receptors for chemotactic factors have been recognized on polymorphonuclear $(\mathrm{PMN})^{1}$ leukocytes by binding studies with radiolabeled synthetic peptides. The binding of the chemotactic factor $\left[{ }^{3} \mathrm{H}\right]-$ formyl-norleucyl-leucyl-phenylalanine to rabbit PMN leukocytes (1) and of $\left[{ }^{3} \mathrm{H}\right]$ formyl-methionyl-leucylphenylalanine to human neutrophils (2) is rapid, saturable and rapidly reversible, and is competitively inhibited by other formylated peptides in a rank-order that reflects their chemotactic potency. The specificity of the putative receptor for formylated peptides was further illustrated by the inhibition of binding of labeled peptide to rabbit PMN leukocytes by small peptides of bacterial origin, whereas comparably chemotactic concentrations of $\mathrm{C} 5 \mathrm{a}$ exhibited no competitive effect (1). Chemotactically inactive analogues, such as carbobenzoxy-phenylalanyl-methionine, bind to the leukocyte receptors for the formylmethionyl peptides and competitively inhibit chemotaxis and other responses elicited by the peptide agonists (3). Of the naturally occurring chemotactic stimuli, only the purified native and synthetic tetrapeptides of the eosinophil chemotactic factor of anaphylaxis have demonstrated a susceptibility to competitive inhibition $(4,5)$. The chemotactically inactive amino-terminal tripeptides reversibly block both the chemotactic and chemotactic-deactivating

\footnotetext{
${ }^{1}$ Abbreviations used in this paper: C5fr, chemotactic fragments of the fifth component of human complement; HBSS, Hanks' balanced salt solution; HBSS-OA, HBSS made 0.4 $\mathrm{g} / 100 \mathrm{ml}$ in ovalbumin; HETE, 12-L-hydroxy-5,8,10,14eicosatetraenoic acid; HHT, 12-L-hydroxy-5,8,10-heptadecatrienoic acid; PMN, polymorphonuclear.
} 
activities of equimolar concentrations of the parent tetrapeptides of eosinophil chemotactic factor of anaphylaxis, without modifying the effects of C5a (4).

The arachidonic acid metabolites, 12-L-hydroxy5,8,10,14-eicosatetraenoic acid (HETE), generated by the action of a lipoxygenase in platelets (6) and some other cells (7), and 12-L-hydroxy-5,8,10-heptadecatrienoic acid (HHT), a cyclo-oxygenase product (8), are chemotactic for PMN leukocytes at concentrations of $0.7-25 \mu \mathrm{g} / \mathrm{ml}$ and $2-50 \mu \mathrm{g} / \mathrm{ml}$, respectively, whereas lower concentrations exhibit chemokinetic activity, and facilitate the responses of PMN leukocytes to other chemotactic factors $(9-11)$. The concentrations of HETE and related fatty acids are strikingly elevated in inflamed tissues of some human diseases, relative to the concentrations in uninvolved tissues, which has suggested an etiological role (12). The observation that the methyl esters of HETE and HHT lacked chemotactic activity and were specific reversible inhibitors of the activity of the native factors, led to a functional analysis of receptor-directed regulation of the in vitro chemotactic responses and the in vivo influxes of leukocytes elicited by the unsaturated hydroxy-fatty acids.

\section{METHODS}

Blind-end acrylic chemotactic chambers with a $0.2-\mathrm{ml}$ stimulus compartment and a $0.5-\mathrm{ml}$ leukocyte well (Neuro Probe, Inc., Bethesda, Md.), 3- $\mu \mathrm{m}$ pore filters (Sartorius, Göttingen, West Germany), Hanks' balanced salt solution without phenol red (Microbiological Associates, Walkersville, Md.), ovalbumin recrystallized five times (Miles Laboratories, Inc., Elkhart, Ind.), dextran, Ficoll-Hypaque, Sephadex G-75 and Sepharose 2B (Pharmacia Fine Chemicals, Inc., Piscataway, N. J.), silica gel $\mathbf{H}$ thin-layer chromatography plates (Analtech, Inc., Newark, Del.), neutral red and fast green (Harleco, American Hospital Supply Corp., Gibbstown, N. J.), Wright-Giemsa stain (Fisher Scientific Co., Pittsburgh, Pa.), zymosan (Sigma Chemical Co., St. Louis, Mo.), arachidonic acid (Nu-Chek-Prep, Elysian, Minn), arachidonate methyl ester and 12-hydroxy stearate methyl ester (Supelco, Inc., Bellefonte, Pa.), indomethacin (Merck Chemical Div. Merck \& Co., Inc. Rahway, N. J.), silicic acid (Silicar CC-4, Mallinckrodt, Inc., St. Louis, Mo.), and $N$-methyl- $N^{\prime}$-nitro$N$-nitrosoguanidine (Aldrich Chemical Co., Inc., Milwaukee, Wis.) were obtained as noted. Rabbit anti-human C5 serum was supplied by Dr. Hans J. Müller-Eberhard (Scripps Clinic and Research Foundation, La Jolla, Calif.). Chemotactic fragments of the fifth component of human complement (C5fr) were prepared with a modification of a method that has been described (13), by incubating $20 \mathrm{ml}$ of fresh serum with $30 \mathrm{mg}$ of zymosan that had been boiled in distilled water and washed twice with Hanks' balanced salt solution. The supernate was titrated to $\mathrm{pH} 5.0$ with $1 \mathrm{M}$ acetic acid, dialyzed

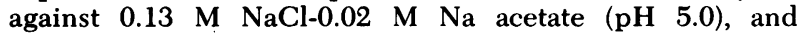
filtered on Sephadex G-75 in the same buffer. The chemotactic activity filtered at $64-72 \%$ bed volume, exhibiting an apparent $16,800 \mathrm{~mol} \mathrm{wt}$, and was pooled and concentrated to $10 \mathrm{ml}$ to serve as a standard preparation of C5fr. Over $90 \%$ of the chemotactic activity was absorbed by Sepharose $2 \mathrm{~B}$ coupled with monospecific rabbit anti-human C5 (14), whereas Sepharose 2B coupled with normal rabbit serum absorbed $<20 \%$ of the activity. Purified synthetic formylmethionyl-phenylalanyl-alanyl-leucine was supplied by Dr. R. J. Freer (Medical College of Virginia, Richmond, Va.). All solvents were either Fisher-certified (Fisher Scientific Co.) and redistilled before use or were obtained from Burdick \& Jackson Laboratories Inc. (Muskegon, Mich.).

Preparation and purification of lipid factors. HETE was prepared by the incubation of arachidonic acid with human platelets in the presence of $0.10 \mathrm{mM}$ indomethacin to block cyclo-oxygenase activity as described (10). HHT was generated by incubating arachidonic acid with a suspension of crude microsomal powder that had been obtained by acetonepentane treatment of sheep seminal vesicles and that served as the source of cyclo-oxygenase $(11,15)$. HETE and HHT were extracted from the respective incubation mixtures and were purified by repeated silicic acid column chromatography (11). The purity of the final products was assessed by trimethylsilyl derivatization and gas-liquid chromatographymass spectrometry $(10,16,17)$; the purity of HETE exceeded $95 \%$ and that of HHT ranged from 85-95\%.

HETE and HHT were methylated with a 100 -fold molar excess of diazomethane that had been generated by the reaction of $30 \mathrm{mg}$ of $N$-methyl- $N^{\prime}$-nitro- $N$-nitrosoguanidine in $0.125 \mathrm{ml}$ of distilled water with $0.15 \mathrm{ml}$ of $5 \mathrm{M} \mathrm{NaOH}$ for $40 \mathrm{~min}$ at room temperature in the inner chamber of a sealed generator that contained $0.75 \mathrm{ml}$ of ether maintained at $4^{\circ} \mathrm{C}$ in the outer jacket to trap the diazomethane (18). A portion of the ethereal solution of diazomethane was added to HETE or HHT in ether, the mixture incubated for $30 \mathrm{~min}$ at room temperature, and the ether evaporated by a stream of nitrogen. The products were redissolved in hexane for storage at $-90^{\circ} \mathrm{C}$. The extent of conversion of HETE and HHT to the corresponding methyl esters was assessed by gas-liquid chromatography of trimethylsilyl derivatives prepared by reaction with trifluorobis (trimethylsilyl) acetamide:trimethylchlorosilane:pyridine (1:1:2, vol:vol:vol) for $30 \mathrm{~min}$ at $65^{\circ} \mathrm{C}$. Portions of the solutions were injected directly into the gas-liquid chromatography apparatus (Hewlett-Packard Co., Palo Alto, Calif., model 5840A) containing $1.8 \mathrm{M}$ glass columns of $3 \% \mathrm{OV}-17$ on Gas-Chrom $\mathrm{Q}$ (Applied Sciences Laboratories Inc., State College, Pa.). The components were eluted by heating the column according to a program that raised the temperature from $100^{\circ} \mathrm{C}$ at a rate of $10^{\circ} \mathrm{C} / \mathrm{min}$. The extent of conversion to the methyl ester exceeded $95 \%$ in three consecutive preparations of HETE and two of HHT, which were used in chemotactic studies without additional purification.

Assessment of in vitro PMN leukocyte chemotaxis and leukocyte influx into the guinea pig peritoneal cavity. Human PMN leukocytes were prepared and purified by centrifugation through Ficoll-Hypaque cushions as described $(19,20)$, and were washed and resuspended to a concentration of $4.2 \pm 0.2 \times 10^{6} / \mathrm{ml}$ in Hanks' balanced salt solution made $0.4 \mathrm{~g} / 100 \mathrm{ml}$ in ovalbumin and $0.005 \mathrm{M}$ in Tris- $\mathrm{HCl}, \mathrm{pH} 7.4$ (HBSS-OA). Chemotactic lipids and methyl ester derivatives were uniformly suspended in HBSS by ultrasonication with six 15-s bursts of $200 \mathrm{~W}$ at $20^{\circ} \mathrm{C}$ with a Branson W140D sonicator (Branson Sonic Power Co., Danbury, Conn.), and the suspensions were diluted in HBSS-OA in the stimulus compartments of the chemotactic chambers. Incubation conditions, processing of filters, and enumeration of PMN leukocytes in stained filters were as detailed (20). Chemotactic responses were expressed as net PMN leukocytes per high-power field after subtraction of the background migration found in chambers lacking a stimulus. The altered chemotaxis observed when a methylated derivative was present in the stimulus compartment was calculated as a percentage of the response to the stimulus alone. 
The in vivo responses to the lipid factors were assessed in groups of three or four 250-g Hartley guinea pigs that had been injected intraperitoneally with $0.4 \mathrm{ml}$ of HBSS containing HETE alone or in combination with methylated HETE. Groups of guinea pigs were sacrificed after $30 \mathrm{~min}$ and $5 \mathrm{~h}$, and the peritoneal cells harvested by washing the peritoneal cavity with three portions of $10 \mathrm{ml}$ of HBSS-OA containing $10 \%$ (vol:vol) $0.15 \mathrm{M}$ citrate anticoagulant (pH 5.2). The washings were pooled, centrifuged at $400 \mathrm{~g}$ for $20 \mathrm{~min}$ at $4^{\circ} \mathrm{C}$, and the cell pellets resuspended in $5 \mathrm{ml}$ HBSS-OA with citrate anticoagulant. Total cell counts were performed by phase-contrast microscopy and replicate smears were prepared for staining with Wright-Giemsa and fast greenneutral red techniques (21). Differential counts of 300 leukocytes/smear demonstrated the presence of macrophages, eosinophils, and neutrophils, as well as occasional other cells that collectively accounted for $<10 \%$ of the total cellular population under all conditions. The number of leukocytes in each class was calculated by multiplying the total number of cells by the percentage of that cell type derived from the differential counts of the fast green-neutral red smears.

\section{RESULTS}

The reaction of HETE and HHT with diazomethane resulted in a $>95 \%$ conversion to the corresponding methyl esters, which was associated with a decrease in their chemotactic activity for human PMN leukocytes. In three experiments, $4 \mu \mathrm{g} / \mathrm{ml}$ and $16 \mu \mathrm{g} / \mathrm{ml}$ of the methyl ester of HETE exhibited chemotactic activity that ranged from 2 to $9 \%$ and 6 to $11 \%$, respectively, of that manifested by the same concentrations of native HETE. Comparable studies with 8 and $32 \mu \mathrm{g} / \mathrm{ml}$ of the methyl ester of HHT revealed residual chemotactic activity of $4-10 \%$ and $5-14 \%$, respectively. As the methyl esters of HETE and HHT demonstrated only marginal chemotactic activity and no detectable chemokinetic activity, they were examined for their capacity to modulate the chemotactic response to the native hydroxy-fatty acids. The addition to the stimulus compartment of progressively greater quantities of HETE methyl ester significantly inhibited the expression of the chemotactic activity of the native principle in a dose-related manner (Fig. 1). Approximately $50 \%$ inhibition of the responses to both $1 \mu \mathrm{g} / \mathrm{ml}$ and $4 \mu \mathrm{g} / \mathrm{ml}$ of HETE was achieved by an equimolar concentration of HETE methyl ester. The chemotactic response to $8 \mu \mathrm{g} / \mathrm{ml}$ of HHT, a stimulus exhibiting one-half to one-third the potency of HETE (11), was inhibited by HETE methyl ester by over $60 \%$ at a molar ratio of 0.25 . In contrast, $1 \mu \mathrm{g} / \mathrm{ml}$ to $16 \mu \mathrm{g} / \mathrm{ml}$ of HETE methyl ester inhibited by $<7 \%$ the chemotaxis to varying concentrations of C5fr and f-Met-Phe-Ala-Leu that elicited responses ranging from 17 to 59 and 21 to 74 net PMN leukocytes per high-power field, respectively. HHT methyl ester inhibited the chemotactic response to HHT with $\cong 50 \%$ inhibition at a molar ratio of 0.5 , but had no effect on the response to C5fr or f-Met-Phe-Ala-Leu (Table I). A relationship reciprocal to that seen for HHT inhibition by HETE

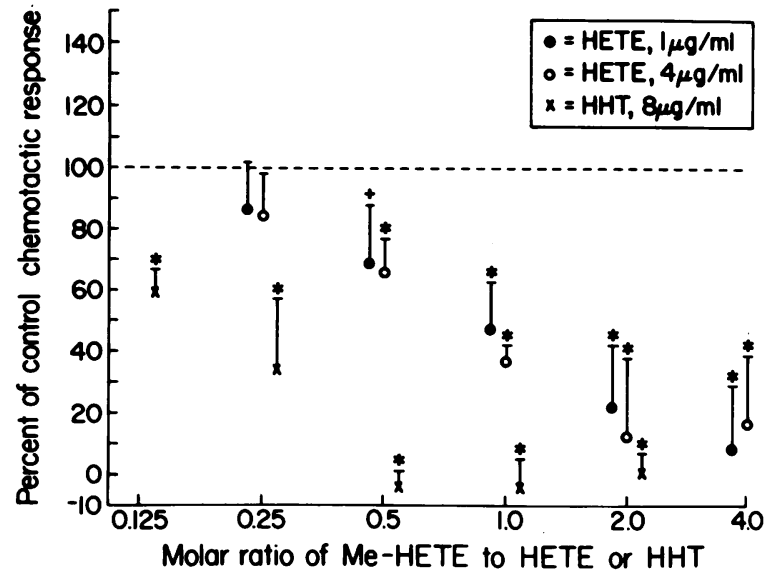

FIGURE 1 Chemotactic inhibitory effect of HETE methyl ester (Me-HETE). Each data point and bracket represent the mean \pm SD for three experiments with PMN leukocytes from separate donors. The control chemotactic responses in the absence of HETE methyl ester $(100 \%)$ were $9.9,14.6$, and 12.3 net PMN leukocytes per high-power field for $1 \mu \mathrm{g}$ $\mathrm{HETE} / \mathrm{ml}, 24.7,20.2$, and 30.3 for $4 \mu \mathrm{g} \mathrm{HETE} / \mathrm{ml}$, and 19.6, 17.4, and 18.9 for $8 \mu \mathrm{g} \mathrm{HHT} / \mathrm{ml}$. Levels of statistical significance are indicated by the symbols + for $P<0.05$ and $*$ for $P<0.01$.

methyl ester was also appreciated, as $50 \%$ inhibition of the chemotaxis elicited by HETE required an eightfold molar excess of HHT methyl ester. The inhibition of the chemotactic response to either native fatty acid by a given concentration of the corresponding methyl ester was greater when the methyl ester was introduced into the cell well than when it was mixed with the stimulus. The possibility of a noncompetitive cell-directed effect was excluded, because the inhibition of chemotaxis produced by adding 8 $-32 \mu \mathrm{g} / \mathrm{ml}$ of each of the methyl esters to portions of PMN leukocytes was eliminated by washing the cells twice in HBSS-OA before assessment of the chemotactic response to the native fatty acids. The specificity of the inhibition by the homologous methyl esters was confirmed by the lack of effect of methyl esters of structurally related, but nonchemotactic, fatty acids (Table II). The methyl ester of 12-hydroxy stearate, which lacks the unsaturation of HETE and HHT, and the methyl ester of arachidonate, which has no 12-hydroxy group, did not inhibit the PMN leukocyte chemotactic response to HETE or HHT at equimolar concentrations. In the same experiments, HETE methyl ester inhibited the chemotactic responses to HETE and HHT by over $50 \%$ at molar ratios of 1 and 0.25 , respectively. The highest concentration of 12-hydroxy stearate methyl ester and arachidonate methyl ester suppressed PMN leukocyte chemotaxis elicited by C5fr, as well as by HETE and HHT, which suggested an inhibitory mechanism that was not related to the receptors for unsaturated hydroxy-fatty acids. 
TABLE I

Chemotactic Inhibitory Effect of HHT Methyl Ester

\begin{tabular}{lcccr}
\hline & \multicolumn{4}{c}{ Concentration of HHT methyl ester, $\mu \mathrm{g} / \mathrm{ml}$} \\
\cline { 2 - 5 } Chemotactic stimulus & 2 & 4 & 8 & 16 \\
\hline HHT $(8 \mu \mathrm{g} / \mathrm{ml})$ & $71 \pm 18^{*}$ & $45 \pm 15$ & $37 \pm 9$ & $4 \pm 11$ \\
HETE $(2 \mu \mathrm{g} / \mathrm{ml})$ & $95 \pm 5$ & $85 \pm 7$ & $72 \pm 13$ & $42 \pm 16$ \\
C5fr $\$$ & $96 \pm 8$ & $104 \pm 10$ & $111 \pm 14$ & $94 \pm 11$ \\
F-Met-Phe-Ala-Leu $(50 \mathrm{nM})$ & - & - & $103 \pm 9$ & $91 \pm 16$ \\
\hline
\end{tabular}

* Each value is the mean \pm 0.5 the range for two experiments performed in duplicate, where the results are expressed as a percentage of the control response to the stimulus in the absence of HHT methyl ester. The mean control responses (100\%) were 23.6 net PMN per high-power field for HHT, 18.2 for HETE, 31.9 for C5fr, and 29.4 for F-Met-Phe-Ala-Leu.

$\$$ C5fr were employed at a dilution of 1:60 of the standard preparation.

Preliminary experiments were carried out to establish the dose relationship and time-course of the cellular response of guinea pigs to intraperitoneally administered HETE. Eosinophils respond earlier than neutrophils with peak levels at $30 \mathrm{~min}$ and $5 \mathrm{~h}$, respectively, and the neutrophils clearly predominated by $5 \mathrm{~h}$. The guinea pig peritoneal cellular response to HETE alone and in combination with varying doses of HETE methyl ester was studied in additional groups of guinea pigs (Fig. 2). At $30 \mathrm{~min}$, significant increases in the number of eosinophils and neutrophils were elicited by 4 and $8 \mu \mathrm{g}$ of HETE, but the eosinophils were the predominant cell type. The eosinophil response to $8 \mu \mathrm{g}$ of HETE was inhibited in a dose-related fashion by equimolar and twofold molar quantities of HETE methyl ester, which elicited no cellular response in its own right. At $5 \mathrm{~h}$, both doses of HETE evoked significant increments in the number of neutrophils while the eosinophils responded slightly only to the lower dose of $4 \mu \mathrm{g}$ of HETE. HETE methyl ester suppressed the neutrophil response to $8 \mu \mathrm{g}$ of HETE at both the equimolar and twofold molar

TABLE II

Effects on Chemotaxis of Nonchemotactic Fatty Acid Methyl Esters and of HETE Methyl Ester

\begin{tabular}{lccccc}
\hline & \multicolumn{5}{c}{ Concentration of 12 -hydroxy stearate methyl ester, $\mu \mathrm{g} / \mathrm{ml}$} \\
\cline { 2 - 6 } Chemotactic stimulus & 1 & 2 & 4 & 8 & 16 \\
\hline HHT & - & $106 \pm 11^{*}$ & $95 \pm 9$ & $79 \pm 6$ & - \\
HETE & $101 \pm 4$ & $93 \pm 4$ & $101 \pm 18$ & $82 \pm 7$ & $51 \pm 8$ \\
C5fr & $114 \pm 10$ & - & $105 \pm 3$ & $86 \pm 12$ & $63 \pm 6$ \\
& & Concentration of arachidonate methyl ester, $\mu \mathrm{g} / \mathrm{ml}$ & \\
\cline { 2 - 6 } & 1 & 2 & 4 & 8 & 16 \\
HHT & - & $96 \pm 9$ & $103 \pm 11$ & $81 \pm 8$ & $58 \pm 10$ \\
HETE & $117 \pm 9$ & $100 \pm 3$ & $89 \pm 5$ & $72 \pm 12$ & $46 \pm 7$ \\
C5fr & $102 \pm 13$ & - & $100 \pm 6$ & $84 \pm 8$ & $60 \pm 5$ \\
& & & & & \\
& & Concentration of & HETE methyl ester, $\mu \mathrm{g} / \mathrm{ml}$ & \\
& 1 & 2 & 4 & 8 & 16 \\
HHT & $65 \pm 14$ & $41 \pm 9$ & $3 \pm 10$ & - & - \\
HETE & $59 \pm 8$ & $34 \pm 6$ & $11 \pm 5$ & $13 \pm 2$ & - \\
C5fr & $127 \pm 19$ & $113 \pm 12$ & $89 \pm 7$ & - & $92 \pm 11$ \\
\hline
\end{tabular}

* Each value is the mean \pm 0.5 the range for two experiments performed in duplicate, where the results are expressed as a percentage of the control response to the stimulus in the absence of a methyl ester. The mean control responses (100\%) were 26.5 net PMN per high-power field for $8 \mu \mathrm{g} / \mathrm{ml}$ of HHT, 24.9 for $2 \mu \mathrm{g} / \mathrm{ml}$ of HETE, and 28.3 for a 1:60 dilution of the standard preparation of C5fr. 


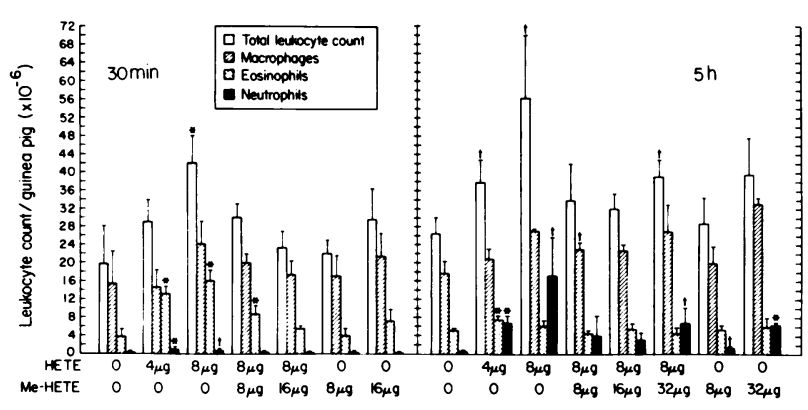

FIGURE 2 In vivo inhibitory effect of HETE methyl ester (Me-HETE) on leukocyte influx into the guinea pig peritoneal cavity. Each bar and bracket represents the mean \pm SD for cell counts from four guinea pigs at the 30-min interval and three guinea pigs at the 5-h interval. The levels of statistical significance are indicated as noted in Fig. 1.

levels, whereas the suppression was less at a fourfold molar excess of HETE methyl ester. The latter dose of HETE methyl ester alone elicited a substantial peritoneal neutrophilia that was comparable to that evoked by $4 \mu \mathrm{g}$ of native HETE.

\section{DISCUSSION}

The ability of the chemotactically inactive methyl esters of HETE and HHT to inhibit the PMN leukocyte migratory responses to the native fatty acids is ascribed to a specific competitive mechanism based on the functional characteristics of the inhibitory effect. The chemotactic activity of HETE and HHT in vitro (Fig. 1, Table I) and the ability of HETE to stimulate leukocyte influx in vivo (Fig. 2), were inhibited by $50 \%$ or more by an equimolar concentration of the respective methyl ester. In crossed inhibition experiments, HETE methyl ester suppressed the chemotactic response to HHT by over $60 \%$ at a molar ratio of 0.25 , whereas HHT methyl ester inhibition of the chemotactic activity of HETE required a molar ratio of $\cong 8$. The results of such reciprocal inhibition studies not only reflect the greater potency of HETE relative to HHT, but suggest that the two factors bind to the same P.MN leukocyte receptors with an affinity that parallels their chemotactic potency. That the inhibition of chemotaxis achieved by adding either methyl ester to the PMN leukocytes was completely reversed by washing the cells before assessing their response to the native fatty acids further suggested a competitive mechanism of action. Finally, the specificity of the competitive effect was demonstrated by the failure of HETE or HHT methyl esters to inhibit comparable chemotactic responses to heterologous stimuli, such as fragments of $\mathrm{C} 5$ and formyl-methionyl peptides, and by the failure of methyl esters of structurally related, but nonchemotactic, fatty acids to inhibit the chemotactic responses to equimolar concentrations of HETE and
HHT (Tables I and II). The inhibition of in vitro and in vivo PMN leukocyte responses to formyl-methionyl peptides by the chemotactically inactive derivative carbobenzoxy-phenylalanyl-methionine, which is an antagonist of the binding of formyl-methionyl peptides to PMN leukocytes, exhibited a specificity analogous to the unsaturated hydroxy-fatty acid methyl esters in that no effect was observed on the response to C5a (3). In this case, however, the competitive mechanism was less apparent as the weak binding of carbobenzoxyphenylalanyl-methionine to the PMN leukocytes necessitated the introduction of concentrations far higher than the agonists to inhibit chemotaxis by $50 \%$; such molar ratios ranged from $10^{2}$ for formyl-methionylmethionyl-methionine to $10^{5}$ for formyl-methionylleucyl-phenylalanine (3). The inhibitory action of carbobenzoxy-phenylalanyl-methionine with respect to other PMN leukocyte-directed activities of the formylmethionyl peptides, including aggregation, release of granular enzymes, and the induction of in vivo neutropenia, required similarly high molar ratios relative to the stimuli. Nonetheless, the present data and that obtained with the formyl-methionyl peptides together suggest the possibility of discrete classes of chemotactic receptors on PMN leukocytes for hydroxyfatty acids, formyl-methionyl peptides, and C5a.

The in vivo activity of HETE exhibited several unique features that were not predictable solely from the available in vitro data. The leukocytes responding to HETE at $30 \mathrm{~min}$ were predominantly eosinophils, which most likely reflects the eosinophil preference of the stimulus (10) as well as the greater immediate supply in the tissues of eosinophils as compared to neutrophils $(22,23)$. The failure of HETE to mobilize an increased number of peritoneal macrophages, probably reflects the relatively weak chemotactic activity of HETE for the mononuclear leukocyte series (10). The in vivo activity for neutrophils expressed at $5 \mathrm{~h}$ by high doses of HETE methyl ester alone partially blunted the inhibition of the response to HETE afforded by lower concentrations of the methyl ester (Fig. 2). Whether the in vivo activity of HETE methyl ester is a reflection of the marginal activity detected in vitro or of in vivo cleavage that liberates HETE must await studies of the in vivo hydrolysis of radiolabeled HETE methyl ester. Nonetheless, the results warrant an examination of the inhibitory effects of other carboxyl derivatives of HETE that are likely to be more resistant to in vivo degradation.

\section{REFERENCES}

1. Aswanikumar, S., B. A. Corcoran, and E. Schiffman. 1977. Demonstration of a receptor on rabbit neutrophils for chemotactic peptides. Biochem. Biophys. Res. Commun. 74: $810-817$.

2. Williams, L. T., R. Snyderman, M. C. Pike, and R. J. 
Leftkowitz. 1977. Specific receptor sites for chemotactic peptides on human polymorphonuclear leukocytes. Proc. Natl. Acad. Sci. U. S. A. 74: 1204-1211.

3. O'Flaherty, J. T., H. J. Showell, D. L. Kreutzer, P. A. Ward, and E. L. Becker. 1978. Inhibition of in vivo and in vitro neutrophil responses to chemotactic factors by a competitive antagonist. J. Immunol. 120: 1326-1340.

4. Goetzl, E. J., and K. F. Austen. 1976. Structural determinants of the eosinophil chemotactic activity of the acidic tetrapeptides of the eosinophil chemotactic factor of anaphylaxis. J. Exp. Med. 144: 1424-1437.

5. Boswell, R. N., E. J. Goetzl, and K. F. Austen. 1976. A chemotactic receptor for Val(Ala)-Gly-Ser-Glu on human eosinophil polymorphonuclear leukocytes. Immunol. Commun. 5: 469-479.

6. Nugteren, D. J. 1975. Arachidonic lipoxygenase in blood platelets. Biochim. Biophys. Acta. 380: 299-307.

7. Roberts, L. J. II, R. A. Lewis, R. Hansbrough, K. F. Austen, and J. A. Oates. 1978. Biosynthesis of prostaglandins, thromboxanes, and 12-L-hydroxy-5,8,10,14-eicosatetraenoic acid by rat mast cells. Fed. Proc. 37: 384A. (Abstr.)

8. Hamberg, M., J. Svensson, and B. Samuelsson. 1974. Prostaglandin endoperoxides. A new concept concerning the mode of action and release of prostaglandins. Proc. Natl. Acad. Sci. U. S. A. 71: 3824-3830.

9. Turner, S. R., J. A. Tainer, and W. S. Lynn. 1975. Biogenesis of chemotactic molecules by the arachidonate lipoxygenase system of platelets. Nature (Lond.). 257: $680-681$.

10. Goetzl, E. J., J. M. Woods, and R. R. Gorman. 1977. Stimulation of human eosinophil and neutrophil polymorphonuclear leukocyte chemotaxis and random migration by 12-L-hydroxy-5,8,10,14-eicosatetraenoic acid. J. Clin. Invest. 59: 179-183.

11. Goetzl, E. J., and R. R. Gorman. 1978. Chemotactic and chemokinetic stimulation of human eosinophil and neutrophil polymorphonuclear leukocytes by $12-\mathrm{L}-\mathrm{OH}-$ 5,8,10-heptadecatrienoic acid (HHT). J. Immunol. 120: $526-531$.

12. Hammerstrom, S., M. Hamberg, B. Samuelsson, E. A. Duell, M. Stawski, and J. J. Voorhees. 1975. Increased concentrations of nonesterified arachidonic acid, 12-L- hydroxy-5,8,10,14-eicosatetraenoic acid, prostaglandin $E_{2}$ and prostaglandin $F_{2 \alpha}$ in epidermis of psoriasis. Proc. Natl. Acad. Sci. U. S. A. 72: 5130-5134.

13. Fernandez, H. N., P. M. Henson, A. Otani, and T. E. Hugli. 1978. Chemotactic response to C3a and C5a. I. Evaluation of C3a and C5a leukotaxis in vitro and under simulated in vivo conditions. J. Immunol. 120: 109-115.

14. Cuatrecasas, P., M. Wilchek, and C. B. Anfinsen. 1968. Selective enzyme purification by affinity chromatography. Proc. Natl. Acad. Sci. U. S. A. 61: 636-643.

15. Wallach, D. P., and E. G. Daniels. 1971. Properties of a novel preparation of prostaglandin synthetase from sheep seminal vesicles. Biochim. Biophys. Acta. 231: 445-453.

16. Hamberg, M., and B. Samuelsson. 1974. Prostaglandin endoperoxides. Novel transformation of arachidonic acid in human platelets. Proc. Natl. Acad. Sci. U. S. A. 71: 3400-3404.

17. McGuire, J. C., R. C. Kelley, R. R. Gorman, and F. F. Sun. 1978. Preparation and spectral properties of 12-hydroxyleicosatetraenoic acid (HETE). Prep. Biochem. 8: 147-153.

18. Fales, H. M., T. M. Jaouni, and J. F. Babashak. 1973. Simple device for preparing ethereal diazomethane without resorting to codistillation. Anal. Chem. 45: 2302-2304.

19. Boyum, A. 1968. Isolation of leukocytes from human blood: Further observations. Scand. J. Lab. Clin. Invest. 21(Suppl. 97): 31-48.

20. Goetzl, E. J., and K. F. Austen. 1972. A neutrophil immobilizing factor derived from human leukocytes. I. Generation and partial characterization. J. Exp. Med. 136: 1564- 1580 .

21. Ottesen, E. A., and S. G. Cohen. 1978. The eosinophil, eosinophilia, and eosinophil related disorders. In Allergy: Principles and Practice. E. Middleton, C. E. Reed, and E. F. Ellis, editors. The C. V. Mosby Co., New York. 584-632.

22. Hudson, G. 1968. Quantitative study of the eosinophil granulocytes. Semin. Hematol. 5: 166-186.

23. Osgood, E. E., A. J. Seaman, J. Tivey, and D. A. Rigas. 1954. Duration of life and of different stages of maturation of normal and leukemic leukocytes. Rev. Hematol. 9: 543-554. 